\title{
SIGN CHANGES IN LINEAR COMBINATIONS OF DERIVATIVES AND CONVOLUTIONS OF POLYA FREQUENCY FUNCTIONS
}

\author{
STEVEN NAHMIAS \\ Department of Mathematics \\ University of Pittsburgh \\ Pittsburgh, Pennsylvania 15213 U.S.A.
}

\section{FRANK PROSCHAN}

Department of Statistics

Florida State University

Tallahassee, Florida 32306 U.S.A.

(Received September 5, 1978)

ABSTRACT. We obtain upper bounds on the number of sign changes of linear combnations of derivatives and convolutions of Fôlya frequency functions using the variation diminishing properties of totally positive functions. These constitute extensions of earlier results of Karlin and Proschan.

KEY WORDS AND PHRASES. Polya Frequency Functions, Sign Changes, Derivatives, Convolutions, Total Positivity, Variation Diminishing Property, Linear Combinations, Sign Regular Functions.

AMS (MOS) SUBJECT CLASSIFICATION (1970) CODES. 34-42. 
1. INTRODUCTION AND SUMMARY. In Karlin and Proschan [1] and Proschan [2] results are obtained concerning the number of sign changes of linear combinations of convolutions of sign regular functions, while Karlin [3, 4, pp. 325-326] has obtained upper bounds on the number of sign changes of linear combinations of first and second derivatives of such functions. (For a function $f$ defined on the real line we denote the number of sign changes of $f$ by $S(f)=\sup S\left[f\left(t_{1}\right), f\left(t_{2}\right), \ldots, f\left(t_{m}\right)\right]$, where the supremum is extended over all sets $t_{1}<t_{2}<\ldots<t_{m}$ on the real line, $m$ is arbitrary but finite, and $S\left(x_{1}, \ldots, x_{m}\right)$ is the number of sign changes of the indicated sequence, zero terms being discarded. For the definition of sign regularity, see Karlin [4, p. 12.] In the present paper, sharper versions of these results are obtained in addition to conclusions regarding linear combinations of both derivatives and convolutions of P6lya frequency functions.

1.1. DEFINITION. A function $f$ defined on the real line is said to be a P6lya frequency function order $n\left(\mathrm{PF}_{n}\right)$ if $x_{1}<\ldots<x_{k}, y_{1}<\ldots<y_{k}$ imply

$$
\left|\begin{array}{ccc}
f\left(x_{1}-y_{1}\right) & \ldots & f\left(x_{1}-y_{k}\right) \\
\cdot & \ldots & \dot{\cdot} \\
f\left(x_{k}-y_{1}\right) & \ldots & f\left(x_{k}-y_{k}\right)
\end{array}\right| \geq 0
$$

for $k=1,2, \ldots, n$.

Note that $\mathrm{PF}_{\mathrm{n}}$ functions possess the sign regularity property.

2. LINEAR COMBINATIONS OF DERIVATIVES OF PÓLYA FREQUENCY FUNCTIONS. The following result is well known (see Karlin, [4], p. 326); but is included since it is the basis for deriving many of our results. A proof is included for completeness and to illustrate our method of approach.

2.1. LEMMA. Assume that $\mathrm{f}$ is a $P F_{n+1}$ function for fixed $n=1,2, \ldots$ Then the 
$n^{\text {th }}$ derivative, $f^{(n)}(x)$, changes sign at most $n$ times. When $n$ sign changes do occur, they occur in the order $+\ldots+\ldots \pm$.

PROOF. Write

$$
\begin{aligned}
f^{(n)}(x) & =\lim _{\Delta \downarrow 0} \frac{1}{\Delta^{n}} \sum_{k=0}^{n}\left(\begin{array}{l}
n \\
k
\end{array}\right)(-1)^{n-k} f(x+k \Delta) \\
& =\lim _{\Delta \downarrow} \frac{1}{\Delta^{n}} \lim _{m \rightarrow \infty} \sum_{k=0}^{n}\left(\begin{array}{l}
n \\
k
\end{array}\right)(-1)^{n-k} \int_{-\infty}^{\infty} g_{m}(k \Delta+u) f(x-u) d u,
\end{aligned}
$$

where

$$
g_{m}(u)=\left\{\begin{array}{l}
m \text { for } 0 \leq u \leq 1 / m \\
0 \text { el sewhere }
\end{array}\right.
$$

Thus

$$
f^{(n)}(x)=\lim _{\Delta \downarrow 0} \frac{1}{\Delta^{n}} \lim _{m \rightarrow \infty} \int_{-\infty}^{\infty}\left[\sum_{k=0}^{n}\left(\begin{array}{l}
n \\
k
\end{array}\right)(-1)^{n-k} g_{m}(k \Delta+u)\right] f(x-u) d u
$$

for fixed $\Delta>0$ and $m$ sufficiently large, the bracketed sum will have sign pattern $+-+\ldots \pm$ (the final sign being + or - as $n$ is odd or even, respectively). No additional sign changes are introduced as $m \rightarrow \infty$ and $\Delta \downarrow 0$. By the variation diminishing property (VDP) of the $\mathrm{PF}_{n+1}$ function $f$ (see Karlin, [4], Chap. 6), the desired result follows. ||

The following result may be established by essentially the same approach as that above and by using the variation diminishing property of PF functions.

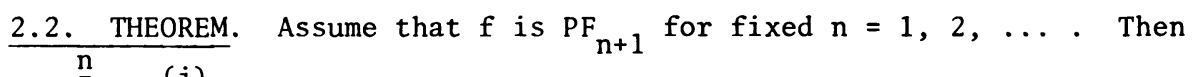
$g(x)=\sum_{j=0}^{n} b_{j} f^{(j)}(x)$ possesses at most $n$ sign changes.

It is interesting to note that Theorem 2.2 can also be obtained by applying known results. From Theorem 2.1 on $p .50$ of $\operatorname{Karlin}[4]$, we find that $\left\{f, f^{(1)}, f^{(2)}, \ldots, f^{(n)}\right\}$ comprises a Weak Tchebychev (WT) system. That the generalized polynomial, $g(x)$, possesses no more than $n$ changes of sign follows from Theorem 4,1 on p, 22 of Karlin and Studden [9].

However, the essential method of proof for Lemma 2,1 may be used to obtain addi= 
tional results which are not a consequence of the theory of WT Systems. In particular we have the following.

2.3. THEOREM. Suppose that $P_{0}, P_{1}, \ldots, P_{n}$ are nonnegative integers, $a_{0}, a_{1}$, $\ldots, a_{n}$ are non-zero real numbers, and $t_{0}<t_{1}<\ldots<t_{n}$ are ordered real numbers. Let $f$ be $P F_{w+1}$, where

$$
\begin{gathered}
w=\sum_{j=0}^{n} P_{j}+\sum_{j=0}^{n-1} U\left(a_{j}, a_{j+1}, P_{j}\right) \text { and } \\
U\left(a_{j}, a_{j+1}, P_{j}\right)=\left\{\begin{array}{c}
1 \text { if } P_{j} \text { is odd and } S\left(a_{j}, a_{j+1}\right)=0 \text {, or } \\
\text { if } P_{j} \text { is even and } S\left(a_{j}, a_{j+1}\right)=1 \\
0 \quad \text { otherwise. }
\end{array}\right.
\end{gathered}
$$

Then $g(x)=\sum_{j=0}^{n} a_{j} f^{\left(P_{j}\right)}\left(x-t_{j}\right)$ possesses at most w sign changes.

PROOF. As in the proof of Lemma 2.1, we may write, after interchanging the integral and the summation sign,

$$
\left.g(x)=\lim _{\Delta \downarrow 0} \lim _{m \rightarrow \infty} \int_{-\infty}^{\infty} \sum_{j=0}^{n}\left(a_{j} / \Delta{ }^{P}\right)\left[\sum_{k=0}^{P_{j}\left(_{k}{ }^{p}\right.}\right)(-1)^{P_{j}-k} g_{m}\left(k \Delta+u-t_{j}\right)\right] \cdot f(x-u) d u,
$$

where $g_{m}(\cdot)$ has the same definition as in the proof of Lemma 2.1 .

As $u$ approaches $t_{j}-k \Delta$, the bracketed term will have sign pattern $+-+\ldots \pm$, the final sign being + if $P_{j}$ is even and - if $P_{j}$ is odd. It follows that for $u$ near $t_{j}-k \Delta$, the term $a_{j} \cdot[]$ will have sign pattern

$$
\begin{aligned}
& +-+\ldots+\text { if } a_{j}>0 \text { and } P_{j} \text { is even } \\
& +-+\ldots-\text { if } a_{j}>0 \text { and } P_{j} \text { is odd } \\
& -+\ldots-\text { if } a_{j}<0 \text { and } P_{j} \text { is even } \\
& -+\ldots+\text { if } a_{j}<0 \text { and } P_{j} \text { is odd. }
\end{aligned}
$$

Each term $a_{j} \cdot\left[\right.$ ] will contribute up to $P_{j}$ sign changes. In addition, there will be one more sign change introduced between the final sign of the string of $P_{j}$ plusses 
and minuses at $t_{j+1}$ for $0 \leq j \leq n+1$ if and only if $a_{j}$ and $a_{j+1}$ have opposite signs for $P_{j}$ even and like signs for $P_{j}$ odd.

The number of sign changes is not increased as $\Delta \downarrow 0$ and $m \rightarrow \infty$. The result follows by the VDP property of the $P_{w+1}$ function $f . \|$

An interesting point to note here is that in general it is not possible to determine $w$ from just the knowledge of $S\left(a_{0}, \ldots, a_{n}\right)$. However if all $P_{j}, 0 \leq j \leq n$, are even numbers (zero included), then it is easy to see that $w=\sum_{j=0}^{n} p_{j}+s\left(a_{0}, \ldots, a_{n}\right)$, while if the $P_{j}$ are odd numbers, then $w=\sum_{j=0}^{n} P_{j}+n-S\left(a_{0}, \ldots, a_{n}\right)$.

\section{LINEAR COMBINATIONS OF CONVOLUTIONS OF POLYA FREQUENCY FUNCTIONS.}

Denote by $f^{n^{*}}(x)$ the $n$-fold convolution of a PF function $f$. We have the following result:

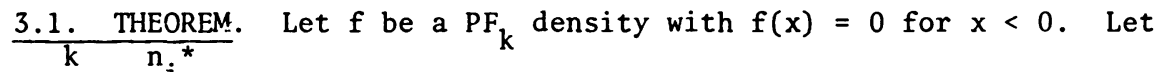
$g(x)=\sum_{i=1}^{k} a_{i} f^{n_{i}{ }^{*}}(x)$, where $n_{1}<n_{2}<\ldots<n_{k}$, and the $a_{i}$ are real non-zero constants. Then $S(g) \leq S(\underline{a})$. Moreover, if $S(g)=S(\underline{a})$, then the sign changes of $\left(a_{1}, \ldots, a_{k}\right)$ and of $\mathrm{g}$ occur in the same order.

PROOF. The proof is by induction on $k$. Clearly the result holds for $k=1$.

Suppose it holds for $1,2, \ldots, k-1$. Then write

$$
g(x)=\sum_{i=1}^{k} a_{i} f^{n_{i}^{*}}(x)=\lim _{m \rightarrow \infty} \int_{0}^{\infty}\left[a_{1} g_{m}(u)+\sum_{i=2}^{k} a_{i} f\left({ }_{i}^{\left(n_{1}-n_{1}\right) *}(u)\right] f^{n_{1}^{*}}(x-u) d u,\right.
$$

where $g_{m}(u)$ is defined in the proof of Lemma 2.1 .

By the inductive hypothesis $s\left(\sum_{i=2}^{k} a_{i} f^{\left(n_{i}-n_{1}\right)^{*}}(u)\right) \leq s\left(a_{2}, \ldots, a_{k}\right)$. Since the term $a_{1} g_{m}(u)$ will introduce no additional sign changes in the bracketed term when $S\left(a_{1}, a_{2}\right)=0$ and $m$ is sufficiently large, and introduces one additional sign change in the bracketed term if $S\left(a_{1}, a_{2}\right)=1$ for $m$ sufficiently large, the result now follows from the variation diminishing property possessed by $f$.

The proof that if $\mathrm{g}$ does possess the full $\mathrm{n}$ sign changes then they occur in 
the same order as in $\left(a_{1}, \ldots, a_{n}\right)$ is simple and so is omitted.

The following result may now be deduced from Theorem 3.1 and the variation diminishing property of $\mathrm{TP}_{\mathrm{k}}$ functions.

3.2. COROLLARY. Let $f$ be $P F_{k}$ and $f(x)=0$ for $x<0$. Then $f^{n^{*}}(x)$ is $T_{k}$ in $\mathrm{n}=1,2, \ldots$ and $\mathrm{x} \geq 0$.

Karlin and Proschan (1960, p. 724) and Proschan (1960, p. 14) obtain a slightly more general version of Corollary 3.2 by using the original definition of totally positive functions. Theorem 3.1 above can then be deduced from the fact that $f^{(n)}(x)$ is $\mathrm{TP}_{\mathrm{k}}$ in $\mathrm{n}$ and $\mathrm{x}$ and the variation diminishing property of $\mathrm{TP}$ functions. We have shown that the same results may be obtained far more simply by proving Theorem 3.1 directly and using the fact that the variation diminishing property characterizes $\mathrm{TP}_{\mathrm{k}}$ functions.

When $f(x)$ does not vanish on the negative half line, a proof similar to that of Theorem 3.1 shows that $\mathrm{g}(\mathrm{x})$ possesses at most $2 \mathrm{~S}(\underline{a})$ changes of sign. This is a sharper version of Theorem 8 on p. 730 of Karlin and Proschan [1] or Theorem 6.1 of Proschan [2].

4. DERIVATIVES AND CONVOLUTIONS. In this section, we extend the results of the previous sections to treat linear combinations of both derivatives and convolutions of P61ya frequency functions.

Although $\left\{f, f^{(1)}, f^{(2)}, \ldots, f^{(n)}\right\}$ constitutes a WT system, one can show that $\left\{f, f^{(1)}, \ldots, f^{(n)}, f^{2^{*}}, \ldots, f^{m^{*}}\right\}$ will not constitute a WT system when $n \geq 1$ and $m \geq 2$. Hence the following is not a consequence of the theory of WT systems.

4.1. THEOREM. Let $1 \leq \mathrm{P}_{0}<\mathrm{P}_{1}<\ldots<\mathrm{P}_{\mathrm{k}}$ and $1<\mathrm{n}_{1}<\ldots<\mathrm{n}_{\mathrm{m}}$ be sequences of nonnegative integers and $a_{1}, \ldots, a_{m}$ and $b_{0}, \ldots, b_{k}$ be sequences of non-zero real numbers. Suppose that $f$ is $P F_{w+1}$ and $f(x)=0$ for $x<0$, where $w=P_{k_{k}}+S(a)+$ $U\left(b_{k}, a_{1}, P_{k}\right)$ and $U$ is defined in Theorem 2.3. Then $g(x)=\sum_{i=1}^{m} a_{i} f^{n_{i}}(x)+\sum_{j=0}^{k} b_{j} f^{\left(P_{j}\right)}(x)$ changes sign at most $w$ times. 
PROOF.

$$
\begin{aligned}
& g(x)=\lim _{\Delta \downarrow 0} \lim _{t \rightarrow \infty} \int_{-\infty}^{+\infty}\left\{\sum_{j=0}^{k} b_{j}\left[\sum_{\ell=0}^{P_{j}} \frac{1}{\Delta P_{j}}\left(\begin{array}{l}
P_{j} \\
j
\end{array}\right){ }_{(-1)}{ }^{P_{j}-\ell} g_{t}(\ell \Delta+u)\right]\right. \\
& \left.+\sum_{i=1}^{m} a_{i} f^{\left(n_{i}-1\right)^{*}}(u)\right\} f(x-u) d u \\
& =\lim _{\Delta \downarrow 0} \lim _{t \rightarrow \infty} \int_{-\infty}^{+\infty}\left\{\sum_{\ell=0}^{P_{k}} w_{\ell}(\Delta) g_{t}(\ell \Delta+u)+\sum_{i=1}^{m} a_{i} f^{\left(n_{i}-1\right)^{*}}(u)\right\} f(x-u) d u,
\end{aligned}
$$

where $w_{\ell}(\Delta)=\sum_{j=a(\ell)}^{k}\left(b_{j} / \Delta P^{P_{j}}\right)\left(\begin{array}{l}P_{j} \\ \ell\end{array}\right)(-1)^{P_{j}^{-\ell}}$ and $a(\ell)=i$ if $P_{i-1}<\ell \leq P_{i}$ for $0 \leq \mathrm{i} \leq \mathrm{k}$ (interpret $\mathrm{P}_{-1}=-1$ ).

For every fixed value of $\Delta>0$, the sequence $\mathrm{w}_{\mathrm{o}}(\Delta), \ldots, \mathrm{W}_{\mathrm{P}_{\mathrm{k}}}(\Delta)$ has at most $P_{k}$ changes of sign (excluding zeros) starting with $\operatorname{sgn}\left(b_{k}\right)$. This sign pattern will occur arbitrarily close to $u=0$ by choosing $\Delta$ sufficiently small and will always dominate the sign of $\sum_{i=1}^{m} a_{i} f^{\left(n_{i}-1\right)^{*}}$ (u) at $u=0$ by choosing $t$ to be sufficiently large.

The term $\sum_{i=1}^{m} a_{i} f^{\left(n_{i}-1\right)^{*}}(u)$ possesses at most $S(\underline{a})$ sign changes as $u$ traverses $\mathrm{R}^{+}$. commencing with $\operatorname{sgn}\left(\mathrm{a}_{1}\right)$. The final sign of the first group of terms will differ from the first sign of the second group if $P_{k}$ is even and $S\left(a_{1}, b_{k}\right)=1$ or if $\mathrm{P}_{\mathrm{k}}$ is odd and $S\left(\mathrm{a}_{1}, \mathrm{~b}_{\mathrm{k}}\right)=0$.

When $f$ does not vanish on the negative half line, we have the following:

4.2. THEOREM. Assume $f$ is $P F_{w+1}$ and $f(x) \neq 0$ for some $x<0$. Suppose that $g(x)$ is as given in Theorem 4.1 and $w=P_{k}+2 s(\underline{a})+c_{k}$, where $c_{k}=1$ if $P_{k}$ is odd and $=2$ if $P_{k}$ is even.

PROOF. As in the proof of Theorem 4.1, the derivative terms in the integrand change sign at most $\mathrm{P}_{k}$ times in a neighborhood around 0 . However, in this case the convolution terms may change sign $2 \mathrm{~S}(\underline{a})$ times. If $\mathrm{P}_{\mathrm{k}}$ is odd then one additional 
sign change is introduced independent of the sign pattern of $\sum_{i=1}^{m} a_{i} f_{i}^{\left(n_{i}-1\right)^{*}}(u)$. However if $\mathrm{P}_{k}$ is even, two additional sign changes may be introduced if the sign of the convolution terms at zero differs from $\operatorname{sgn}\left(b_{k}\right) \cdot \|$

Using essentially the same technique we could determine the maximum number of sign changes of an expression of the form

$$
g(x)=\sum_{j=0}^{n} a_{j} f^{\left(P_{j}\right)}\left(x-t_{j}\right)+\sum_{i=1}^{m} b_{i} f^{k}{ }^{*}\left(x-y_{i}\right)
$$

where $t_{0}<t_{1}<\ldots<t_{n}$ and $y_{1}<y_{2}<\ldots<y_{m}$. The exact upper bound will depend upon the relative magnitudes of the $t_{j}$ 's and $y_{i}$ 's and the sign patterns of $\left(a_{0}, \ldots, a_{n}\right)$ and $\left(b_{1}, \ldots, b_{m}\right)$.

Define $h(x, n, p)=\frac{d^{p}}{d x^{p}} f^{n^{*}}(x)$. Then we have the following result which is similar to Theorem 2.3 .

4.3. THEOREM. Suppose that $\mathrm{P}_{1}, \ldots, \mathrm{P}_{\mathrm{k}}$ are non-negative integers, $a_{1}, \ldots, a_{k}$ are non-zero real numbers, and $n_{1}<n_{2}<\ldots<n_{k}$ is a sequence of increasing positive integers. Let $f$ be $P F_{w+1}$ for

$$
w=\sum_{j=1}^{k} P_{j}+\sum_{j=1}^{k-1} U\left(a_{j}, a_{j+1}, P_{j}\right)
$$

where $U\left(a_{j}, a_{j+1}, a_{i}\right)$ is as defined in Theorem 2.3. Assume that $f(x)=0$ for $x<0$. Then $g(x)=\sum_{j=1}^{k} a_{j} h\left(x, n_{j}, p_{j}\right)$ possesses at most $w$ sign changes commencing with $\operatorname{sgn}\left(a_{1}\right)$.

PROOF. The proof is by induction on $k$. For $k=1$ the result follows from Lemma 2.1 and the fact that the variation diminishing property is possessed by the convolution of PF functions.

Suppose now the result holds for $1,2, \ldots, k-1$. Then 


$$
\begin{aligned}
g(x) & =\lim _{\Delta+0} \lim _{m \rightarrow \infty} \int_{-\infty}^{+\infty}\left\{\left(a_{1} / \Delta^{P}{ }^{\prime}\right) \cdot \sum_{i=0}^{P}\left(\begin{array}{l}
P_{i} \\
i
\end{array}\right)(-1)^{P_{1}-i} g_{m}(i \Delta+u)\right. \\
& \left.+\sum_{j=2}^{k} a_{j} h\left(u, n_{j}-n_{1}, P_{j}\right)\right\} f^{n^{*}}(x-u) d u .
\end{aligned}
$$

The term multiplied by $a_{1}$ will have $P_{1}$ sign changes starting with $\operatorname{sgn}\left(a_{1}\right)$ at $\mathrm{u}_{\mathrm{k}-1}-\mathrm{P}_{1} \Delta, \mathrm{u}=-\left(\mathrm{P}_{1}-1\right) \Delta, \ldots, \mathrm{u}=0$. The remaining terms will have $\sum_{j=2}^{k} P_{j}+$ $\sum_{j=2}^{k-1} U\left(a_{j}, a_{j} a_{j+1}, P_{j}\right)$ sign changes introduced if $S\left(a_{1}, a_{2}\right)=0$ and $P_{1}$ is odd or if $S\left(a_{1}, a_{2}\right)=1$ and $P_{1}$ is even.

4.4. THEOREM. Assume that the vectors $\underline{\mathrm{P}}, \underline{\mathrm{a}}$, and $\underline{\mathrm{n}}$ are as given in Theorem 4.3 except that $f(x) \neq 0$ for some $x<0$. Let $f$ be $P F_{w+1}$ for $w=\sum_{j=1}^{k} P_{j}+\sum_{j=1}^{k-1} c_{j}$, where $c_{j_{k}}$ is defined in Theorem 4.2 and $\sum_{j=1}^{\circ} c_{j} \equiv 0$. Then $g(x)=\sum_{j=1} a_{j} h\left(x, n_{j}, P_{j}\right)$ changes sign at most $w$ times.

PROOF. The result evidently holds for $k=1$.

Assume it holds for argument $1,2, \ldots, k-1$. Then $g(x)$ may be expressed in the same manner as in the proof of Theorem 4.3 except that $h\left(u, n_{j}-n_{1}, P_{j}\right)$ does not vanish for $u<0$. When $P_{1}$ is odd, the first set of terms has sign pattern $+-\ldots$ - for $a_{1}>0$, and $-+\ldots+$ for $a_{1}<0$ which occurs arbitrarily close to $u=0$. In either case the second group of terms introduce exactly one additional sign change. Since by induction the second term has $\sum_{j=2}^{k} P_{j}+\sum_{j=2}^{k-1} C_{j}$ and $P_{1}+1$ additional changes are introduced, the result follows. When $P_{1}$ is even the first group of terms has sign pattern $+\ldots \ldots+$ or $-+\ldots+-$ In either case the second group of terms may introduce two additional sign changes if their sign at zero is or + respectively. ||

Note that when $f(x)$ is a probability density that vanishes on the negative half line, $f^{(j)}(x)$ will possess the full $j$ changes of sign. (See Karlin, [4], p. 326.) 
Hence many of our results hold with equality, and thus we obtain the number of roots of $g(x)$.

In order to illustrate in a simple example how these results might be used, we consider an approximate perishable inventory model developed by Nahmias [6] for a product with a lifetime of m periods, he obtains

$$
w(z)=(1-\alpha) c z+L(z)+(\theta+\alpha c) H(z)-\alpha(\theta+c) \int_{0}^{z} H(z-t) f(d) d t
$$

as the approximate expected cost of ordering to $z$ in each period, where $\alpha=$ the discount factor, $c=$ ordering cost per unit, $L(z)=$ expected holding and shortage cost when ordering to $z, \theta=$ outdate cost, $H(t)=\int_{0}^{t} F^{n^{*}}(u) d u, f(t)=$ one period demand density. When all costs are linear, it follows that

$$
W^{\prime \prime}(z)=(h+r) f(z)+(\theta+\alpha c) f^{m^{*}}(z)-\alpha(\theta+c) f^{(m+1) *}(z),
$$

where $h$ and $r$ are the unit holding and shortage costs respectively. From Theorem 3.1, we see that if $f$ is $\mathrm{PF}_{2}$, then $w^{\prime \prime}(z)$ changes sign no more than once in the order +- . Along with the fact that $w^{\prime}(z)$ changes sign once from - to + (as is demonstrated in Nahmias, [6], this guarantees that the $z^{*}$ minimizing $w(z)$ is the zero of $w^{\prime}(z)$ and that this zero can be found efficiently. As a further description of the behavior of $w$, Theorem 4.3 shows that $w^{\prime \prime \prime}(z)$ changes sign at most four times.

ACKNOWLEDGEMENT: Research supported by National Science Foundation Grant ENG 75-04990 and Air Force Office of Scientific Research, AFSC, USAF, under Grant AFOSR $78-3678$. 


\section{REFERENCES}

[1] Karlin, S. and Proschan, F. Polya type distributions of convolutions. Ann. Math. Statist. 31, (1960). 721-736.

[2] Proschan, F. P6lya Type Distributions in Renewal Theory, With an Application to an Inventory Problem, Prentice-Ha11, Inc, Englewood Cliffs, New Jersey, (1960).

[3] Karlin, S. On games described by bell-shaped kernels. Contributions to the Theory of Games, III (Ann. Math. Studies, Vo1. 39). Princeton University Press, Princeton, N.J., (1957).

[4] Karlin, S. Total Positivity, Vol. 1, Stanford University Press, Stanford, California, (1968).

[5] Karlin, S. and Studden, W. Tchebycheff Systems: With Applications in Analysis and Statistics, John Wiley, New York, (1966).

[6] Nahmias, S. Myopic approximations for the perishable inventory problem. Man. Sci. 22, (1976), 1002-1008. 


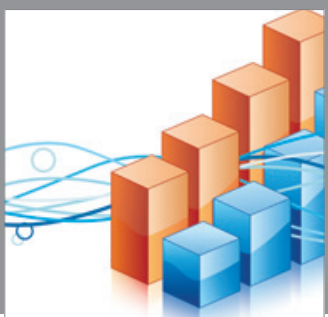

Advances in

Operations Research

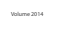

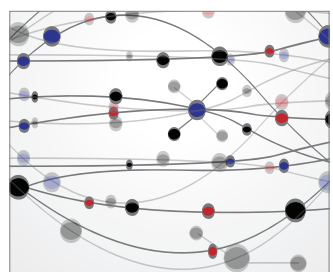

\section{The Scientific} World Journal
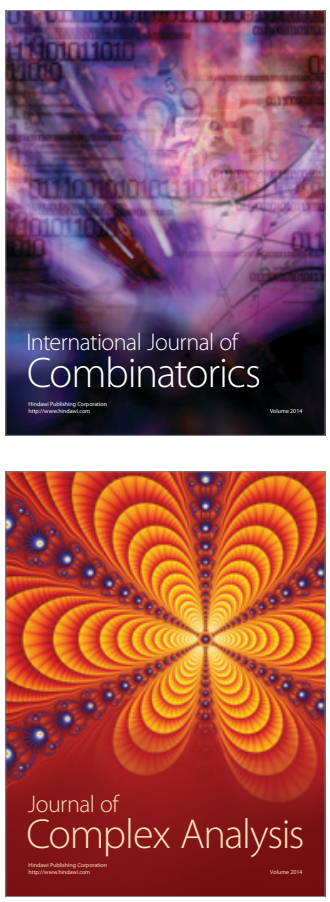

International Journal of

Mathematics and

Mathematical

Sciences
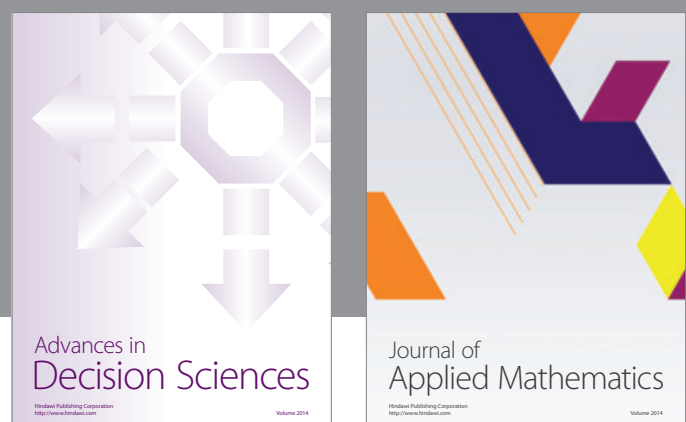

Journal of

Applied Mathematics
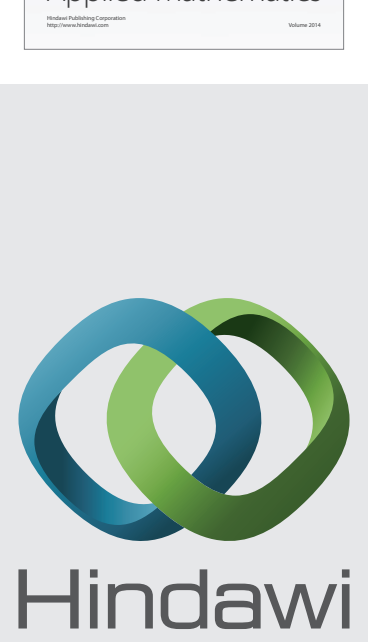

Submit your manuscripts at http://www.hindawi.com
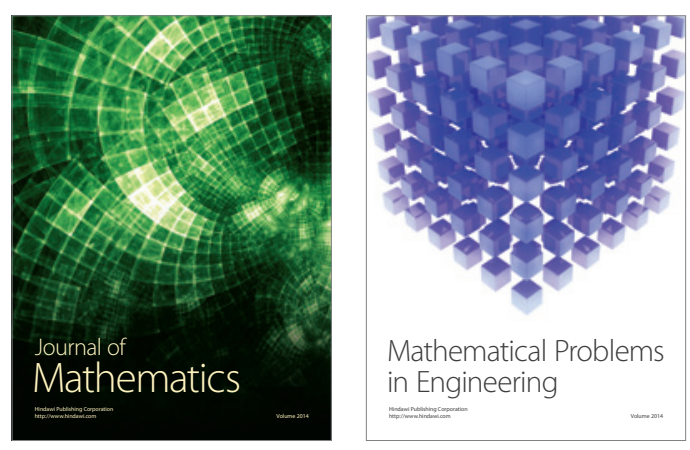

Mathematical Problems in Engineering
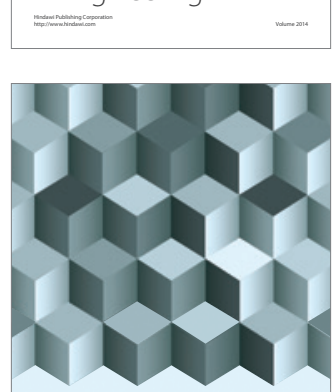

Journal of

Function Spaces
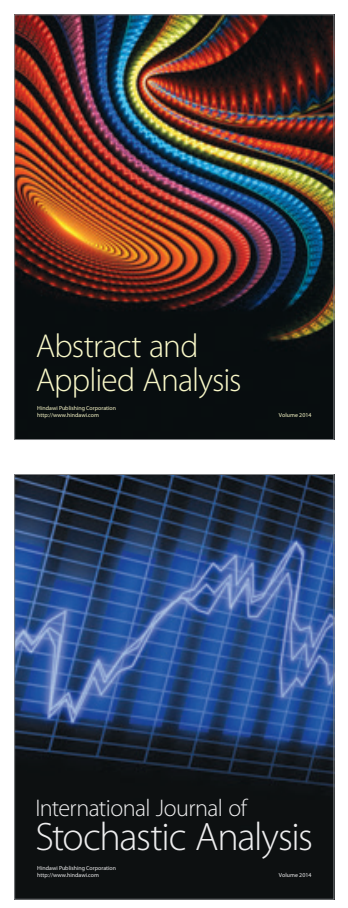

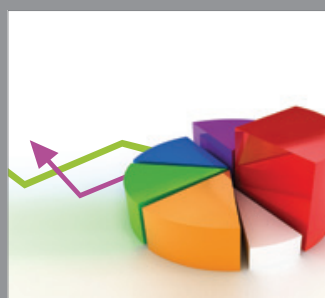

ournal of

Probability and Statistics

Promensencen
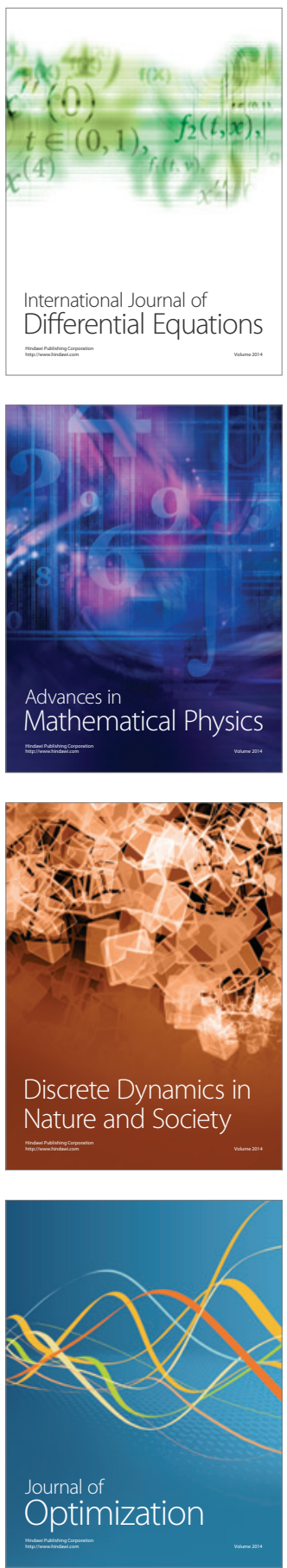